\title{
Prescription profile of Chinese herbal products containing coumestrol, genestein, and/or daidzein among female users: an analysis of national health insurance data in Taiwan between 1997 and 2007
}

\author{
Chien-Tung $\mathrm{Wu}^{1,2}$, Jeng-Nan Tzeng ${ }^{3}$, Jung-Nien Lai ${ }^{2,4^{*}}$, Shun-Hua Tsan ${ }^{5}$ and Jung-Der Wang ${ }^{6,7}$
}

\begin{abstract}
Background: Some Chinese herbs contain several kinds of phytoestrogens, and these herbs are commonly prescribed in Taiwan. Phytoestrogens may influence the effects of estrogen in females, although their activities are weak. This study aims to identify the risk and analyze the prescription profile of commonly used phytoestrogenic herbs in Taiwan.

Methods: The study analyzed women who had been prescribed phytoestrogenic herbs including coumestrol, genistein and/or daidzein between 1997 and 2007 in a fixed cohort taken from all female beneficiaries from the National Health Insurance Research Database of Taiwan. The prescription frequencies, cumulated dosages, and primary indications were listed.

Results: A total of 462,861 women were included in the study, of whom $\sim 47.0 \%$ had used phytoestrogenic herbs at least once during the study period. A total of 6,270,813 prescriptions were recorded, and more than $20 \%$ of these contained phytoestrogens. The most commonly prescribed herb and formula were Puerariae Radix and Ge gen tang (Pueraria Decoction), respectively. Most of the prescriptions were issued for diseases of the respiratory system, followed by symptoms, signs, and ill-defined conditions and diseases of the musculoskeletal system and connective tissue.

Conclusion: This study shows that women who sought medical treatment from Chinese medicine doctors for relief of respiratory discomfort had a high possibility of exposure to phytoestrogenic herbs. Safety issues related to the female endocrine system should be a priority for future research.
\end{abstract}

\section{Background}

Phytoestrogens may interact with the estrogen receptors that mediate endocrine homeostasis, causing damage to reproductive health, especially at developing life stages [1-3]. Although the phytoestrogenic activities are weaker than those of human endogenous estrogens, the consumption of phytoestrogens may have clinically significant

\footnotetext{
* Correspondence: kareny@ms10.hinet.net

${ }^{2}$ Institute of Traditional Medicine, School of Medicine, National Yang-Ming University, Taipei, Taiwan

${ }^{4}$ Department of Obstetrics and Gynecology and Department of Chinese Medicine, Yangming Branch, Taipei City Hospital, Taipei, Taiwan

Full list of author information is available at the end of the article
}

consequences [4]. Coumestrol, genistein and daidzein are strong phytoestrogens that are found in many foods and Chinese medicine $(\mathrm{CM})$ product [5-7]. There is a concern among $\mathrm{CM}$ doctors and $\mathrm{CM}$ users about inconclusive safety results from various in vitro and in vivo models in estrogenic studies, especially for patients taking Chinese herbal products (CHPs) containing coumestrol, genistein, and/or daidzein (CGD-CHPs).

CM has been an important part of healthcare in Taiwan for hundreds of years, and CHPs have been regularly reimbursed by the National Health Insurance (NHI) system since 1995 [8]. All CHPs are fully reimbursed under

\section{Biomed Central}


the current NHI system of Taiwan, and the claim database provides a platform for understanding the utilization of CGD-CHPs.

This study aims to analyze random samples from the NHI database to determine the risk and prescription profile of CGD-CHPs among female beneficiaries in Taiwan between January 1997 and December 2007.

\section{Methods}

\section{Study subjects}

The source population for this study was women who had been prescribed CGD-CHPs between 1997 and 2007 in a randomly sampled cohort from all NHI female beneficiaries in Taiwan. This research included females from the randomly sampled cohort aged 1 to 99 years as the study subjects, and age was calculated by subtracting the subject's birthday from the $1^{\text {st }}$ of July of each year. The NHI system provides universal health insurance coverage, and covered more than $97 \%$ of the Taiwanese population in 2002 [9]. Consequently, data from the NHI system are widely used by researchers in various fields [10]. Both CM and Western medicine doctors must follow a standard diagnostic procedure using the International Classification of Diseases, 9th Revision, Clinical Modification (ICD-9-CM) coding system for claiming reimbursement. Because the NHI system of Taiwan does not reimburse the use of CM for inpatient services, we only investigated the use of $\mathrm{CM}$ for outpatient services. The NHI reimbursement data files were transformed into a research database entitled the National Health Insurance Research Database (NHIRD) that was maintained by the National Health Research Institutes. In the NHIRD, all the identification information is encrypted but the medical records files are retained, including information from 1997 to 2007 on medical care facilities and specialties, drugs and/or treatment regimens (dosages, dosage frequency and prescription duration), patient sex and date of birth, date of visit, and three major diagnoses coded in the ICD-9-CM format. The NHIRD contains a onemillion cohort systematically and randomly selected from the 23 million people in the NHI database who were ever insured under the NHI system in 2005. For each subject, we collected all records of CHPs during 1997 to 2007 as a female cohort. We considered the first diagnosis to be the major diagnosis on the records for outpatient department visits, which were coded in ICD-9-CM and then lumped together into different broader disease categories, e.g., lists of ICD9-CM codes 710-739 were classified as diseases of the musculoskeletal system and connective tissue, while ICD-9-CM codes 460-519 were classified as diseases of the respiratory system.

\section{List of licensed CHPs}

The list of reimbursed CHPs was obtained from the Bureau of the NHI website. The corresponding drug information about specific mixtures or names was obtained from the Committee on Chinese Medicine and Pharmacy (CCMP) website, and included the proportions of each constituent, date, approval period as a drug, code and manufacturers' names. The CCMP list shows that 10,413 CHPs were licensed during the study period, of which 10,309 CHPs were covered by the NHI system. All CHPs with the same CCMP standard formula were classified under the same category, regardless of slight variations among products of different pharmaceutical companies [11]. For example, there are 62 approved licenses for the formula Ge gen tang containing coumestrol, genistein and/or daidzein.

\section{Selection of herbs and estimation of cumulative doses}

The CGD-CHPs examined in this study included Psoraleae Fructus, Puerariae Radix, Sojae Semen Praeparatum, Sophorae Flavescentis Radix and Sophorae Flos. We examined all of the CHPs licensed by the CCMP between 1997 and 2007, including single herbs and herbal formulae, to determine whether they included CGDCHPs. The original weight of each herb was then calculated according to the contents manufactured by the individual pharmaceutical company registered for each licence and approved by the CCMP.

\section{Statistical analysis}

The data were analyzed by descriptive statistics, including the decomposition of the coumestrol, genistein and daidzein contents of the licensed and prescribed CHPs, CGD-CHP prescription rates stratified by age, the medians (with 10th and 90th percentiles) of the cumulative doses of coumestrol, genistein and daidzein, population distribution of patients who had been potentially exposed to coumestrol, genistein and daidzein under various dosages, the frequencies of the disease categories prescribed with CGD-CHPs, the most frequently prescribed herbal formulae containing CGDCHPs, and so on. All of these analyses were performed using the SAS software package (version 9.1; SAS Inc., Cary, NC, USA).

\section{Results}

Between 1 January 1997 and 31 December 2007, 721 (7\%) CGD-CHPs were identified among the 10,309 licensed CHPs, of which the top three CGD-CHPs were Puerariae Radix (4.9\%), Sophorae Flavescentis Radix (0.7\%), and Sojae Semen Praeparatum (0.7\%). A total of $6,270,813$ cases of prescribed and reimbursed CGDCHPs were recorded (Table 1). Among all the CGDCHPs, Puerariae Radix was the most frequently 
Table 1 Distribution frequencies of licensed and prescribed CHPs containing phytoestrogens* based on a one-million patient random sample (1997-2007) from the NHI system of Taiwan

\begin{tabular}{|c|c|c|c|c|c|}
\hline Chinese herbal products & $\begin{array}{l}\text { No. (\%) of } \\
\text { licensed } \\
\text { CHPs }\end{array}$ & $\begin{array}{l}\text { No. (\%) of } \\
\text { female CHP } \\
\text { users }\end{array}$ & $\begin{array}{l}\text { No. (\%) } \\
\text { prescriptions } \\
\text { of CHPs }\end{array}$ & $\begin{array}{l}\text { Cumulative } \\
\text { dose }(g) \text { median } \\
(10 \%-90 \%) \\
\end{array}$ & $\begin{array}{l}\text { Major corresponding } \\
\text { (metabolic) active } \\
\text { substance(s) in humans }\end{array}$ \\
\hline Total no. (\%) licensed CHPs & $10,309(100)$ & $342,440(100)$ & $6,270,813(100)$ & - & \\
\hline Licensed CGD-CHPs & $721(7.0)$ & $217,897(63.6)$ & $1,389,244(22.2)$ & - & \\
\hline Puerariae Fructus & $60(0.6)$ & $13,761(4.0)$ & $35245(0.6)$ & $5.9(2.0-34.0)$ & $\begin{array}{l}\text { Genistein, daidzein and } \\
\text { biochanin A, coumestrol }\end{array}$ \\
\hline Puerariae Radix & $505(4.9)$ & $182,899(53.4)$ & $986,110(15.7)$ & $10.8(1.7-57.6)$ & $\begin{array}{l}\text { Puerarin, daidzin, genistin, } \\
\text { daidzein and genistein, } \\
\text { coumestrol }\end{array}$ \\
\hline Sojae Semen Praeparatum & $74(0.7)$ & $86,273(25.2)$ & $323,413(5.2)$ & $4.2(0.6-17.5)$ & $\begin{array}{l}\text { Genistein, genistin, daidzein, } \\
\text { daidzin, glycitein and glycitin, } \\
\text { coumestrol }\end{array}$ \\
\hline Sophorae Flavescentis Radix & $75(0.7)$ & $63,511(18.5)$ & $180,720(2.9)$ & $4.9(1.5-15.0)$ & $\begin{array}{l}\text { Daidzein, genistein and } \\
\text { formononetin }\end{array}$ \\
\hline Sophorae Flos & $38(0.4)$ & $8,853(2.6)$ & $25,871(0.4)$ & $12.6(2.0-48.0)$ & Genistin, genistein \\
\hline
\end{tabular}

*Phytoestrogens mainly contained coumestrol, genistein and/or daidzein.

prescribed as a single herb and in the formulae by CM practitioners in Taiwan. The co-existence of more than two major active phytoestrogens was identified in licensed and prescribed CGD-CHPs. Genisteincontaining herbal prescriptions comprised $80 \%$ of the prescribed CGD-CHPs.

Among the one-million randomly sampled cohorts, 462,861 women between the ages of 1 and 99 years were included in the analysis. From 1997 to 2007, $342,440(74.0 \%)$ of the women used CHPs on at least one occasion, of whom 217,897 (63.6\%) were treated with prescriptions containing coumestrol, genistein and/or daidzein. The CGD-CHPs-exposed population included many women of reproductive age (Table 2). When calculating individuals' exposure, most patients were exposed to low cumulative doses (less than $50 \mathrm{~g}$ ) of CGD-CHPs, although $5 \%$ of the patients were prescribed with Puerariae Radix at cumulative doses above $57.6 \mathrm{~g}$ (Table 1). Given that the random sample in this cohort accounted for approximately $5 \%$ of the Taiwanese population, it can be inferred that about 180,000 women were exposed to such high cumulative doses of Puerariae Radix.

The major disease categories with CGD-CHPs prescription included respiratory diseases (556,464 visits) and symptoms, signs, and ill-defined conditions $(263,742$ visits), followed by musculoskeletal system and connective tissue diseases (152,471 visits) and digestive diseases (105,448 visits) (Table 3$)$. CGD-CHPs were usually prescribed in the form of herbal formulae, especially Ge gen tang (Pueraria Decoction, containing Puerariae Radix) and Yin qiao san (Lonicera and Forsythia Powder,

Table 2 Prescription frequencies of CHPs containing phytoestrogen ${ }^{a *}$ and cumulative average original weights of the five major Chinese herbs stratified by age, based on a one-million patient random sample (1997-2007) from the NHI system of Taiwan

\begin{tabular}{|c|c|c|c|c|c|c|c|c|c|}
\hline & & & & & Age & & & & \\
\hline Chinese herbal products & $\leq 9$ & 10-19 & $20-29$ & $30-39$ & $40-49$ & $50-59$ & $60-69$ & $70-79$ & $\geq 80$ \\
\hline Total licensed CHPs & 252,268 & 594,059 & $1,069,122$ & $1,368,722$ & $1,332,924$ & 818,922 & 496,445 & 268,747 & 69,604 \\
\hline Licensed CGD-CHPs. & 62,140 & 135,716 & 220,967 & 307,731 & 301,586 & 186,354 & 108,348 & 54,060 & 12,342 \\
\hline \multicolumn{10}{|c|}{ Average dosage per woman-year $(\mathbf{g})^{a}$} \\
\hline Puerariae Fructus & 10.2 & 18.0 & 14.5 & 15.9 & 17.9 & 21.3 & 21.0 & 22.0 & 21.3 \\
\hline Puerariae Radix & 25.8 & 27.8 & 29.2 & 33.7 & 37.0 & 39.0 & 41.4 & 41.5 & 40.4 \\
\hline Sojae Semen Praeparatum & 8.6 & 9.5 & 9.6 & 11.0 & 11.4 & 11.4 & 12.4 & 11.3 & 10.5 \\
\hline Sophorae Flavescentis Radix & 9.0 & 10.5 & 10.9 & 10.9 & 10.7 & 10.5 & 10.8 & 10.6 & 11.4 \\
\hline Sophorae Flos & 15.9 & 24.6 & 27.3 & 33.5 & 35.5 & 37.3 & 36.4 & 49.9 & 34.3 \\
\hline
\end{tabular}

a Converted back to the original weight.

*Phytoestrogens mainly contained coumestrol, genistein and/or daidzein. 
Table 3 Frequency distribution of the top 10 disease categories for prescribing CHPs containing phytoestrogens*

\begin{tabular}{|c|c|c|c|}
\hline Major disease category & ICD-9-CM code range & Number of visits & $\%$ \\
\hline Total & - & $1,389,244$ & 100 \\
\hline Diseases of the respiratory system & $460-519$ & 556,464 & 40.1 \\
\hline Symptoms, signs, and ill-defined conditions & 780-799 & 263,742 & 19.0 \\
\hline Diseases of the musculoskeletal system and connective tissue & 710-739 & 152,471 & 11.0 \\
\hline Diseases of the digestive system & $520-579$ & 105,448 & 7.6 \\
\hline Diseases of the skin and subcutaneous tissue & 680-709 & 95,315 & 6.9 \\
\hline Diseases of the genitourinary system & $580-629$ & 65,837 & 4.7 \\
\hline Injury and poisoning & 800-999 & 39,366 & 2.8 \\
\hline Diseases of the nervous system and sense organs & $320-389$ & 35,585 & 2.6 \\
\hline Diseases of the circulatory system & $390-459$ & 22,932 & 1.7 \\
\hline Endocrine, nutritional and metabolic diseases, and immunity disorders & $240-279$ & 14,889 & 1.1 \\
\hline
\end{tabular}

The population was women aged 0-99 years among the one-million patient NHIRD cohort.

*Phytoestrogens mainly contained coumestrol, genistein and/or daidzein.

containing Sojae Semen Praeparatum) (Table 4). Most CGD-CHPs were prescribed for a treatment period of less than 30 days, and the most common dosage frequency was three times per day. Furthermore, most $\mathrm{CM}$ female users consumed no more than $30 \mathrm{~g}$ of CDGCHPs containing daidzein and genistein.

\section{Discussion}

To the best of our knowledge, this is the first study to document the use of CHPs containing phytoestrogens in a random national-level sample containing all detailed medical records. We focused on active ingredients that could be considered to be potent phytoestrogens, namely coumestrol, genistein and daidzein, in women who sought medical treatment from CM doctors. Among the licensed CHPs in Taiwan, 7\% contained coumestrol, genistein and/ or daidzein. However, these products comprised $22.2 \%$ of the $\mathrm{CM}$ prescriptions consumed by about half of the female population in Taiwan during the 11-year study period. Diseases of the respiratory system were

Table 4 Commonly prescribed single herbs and herbal formulae of CGD-CHPs ${ }^{a}$

\begin{tabular}{|c|c|c|c|c|}
\hline \multicolumn{2}{|l|}{ Drug name } & Herbs with phytoestrogens & Prescriptions & $\%$ \\
\hline \multicolumn{2}{|l|}{ Total herbal prescription } & & $6,270,813$ & 100 \\
\hline \multicolumn{2}{|c|}{ Herbal prescription containing coumestrol, genistein and/or daidzein } & & $1,389,244$ & 22.2 \\
\hline \multicolumn{5}{|l|}{ Single herb (Chinese name) } \\
\hline \multicolumn{2}{|l|}{ Ge Gen } & Puerariae Radix & 223,313 & 3.6 \\
\hline \multicolumn{2}{|l|}{ Bu Gu Zhi } & Puerariae Fructus & 24,040 & 0.4 \\
\hline \multicolumn{2}{|l|}{ Ku Shen Gen } & Sophorae Flavescentis Radix & 17,865 & 0.3 \\
\hline \multicolumn{2}{|l|}{ Huai Hua } & Sophorae Flos & 11,811 & 0.2 \\
\hline \multicolumn{2}{|l|}{ Dan Dou Chi } & Sojae Semen Praeparatum & 5,132 & 0.1 \\
\hline Herbal formulae & (English name) & & & \\
\hline Ge gen tang & Pueraria Decoction & Puerariae Radix & 315,781 & 5.0 \\
\hline Yin qiao san & Lonicera and Forsythia Powder & Sojae Semen Praeparatum & 307,692 & 4.9 \\
\hline Chai ge jie ji tang & Bupleurum and Pueraria Flesh-Resolving Decoction & Puerariae Radix & 115,629 & 1.8 \\
\hline Xiao feng san & Wind-Dispersing Powder & Sophorae Flavescentis Radix & 85,502 & 1.4 \\
\hline Dang gui nian tong tang & Chinese Angelica Pain-Assuaging Decoction & Puerariae Radix Sophorae Flavescentis Radix & 81,063 & 1.3 \\
\hline Shi shen tang & Ten Spirits Decoction & Puerariae Radix & 75,271 & 1.2 \\
\hline Ge gen gin lian tang & Pueraria, Scutellaria, and Coptis Decoction & Puerariae Radix & 60,686 & 1.0 \\
\hline Shen su yin & Ginseng and Perilla Beverage & Puerariae Radix & 34,848 & 0.6 \\
\hline Qing shu yi qi tang & Summerheat-Clearing Qi-Boosting Decoction & Puerariae Radix & 32,338 & 0.5 \\
\hline San zhong kui jian tang & Swelling-Dispersing Hardness-Breaking Decoction & Puerariae Radix & 27,071 & 0.4 \\
\hline
\end{tabular}

${ }^{a}$ CGD-CHPs refers to Chinese herbal products containing coumestrol, genistein and/or daidzein. 
the most frequent disease category for which CGDCHPs were prescribed, constituting $40 \%$ of $\mathrm{CM}$ visits. This finding indicates that female $\mathrm{CM}$ users in Taiwan may not have expected to be exposed to phytoestrogenic herbs when they used CM therapies to manage the discomfort of a sore throat, cough, and runny nose. Exposure to Puerariae Radix was the most extensive, and nearly $5 \%$ of female CM users consumed cumulative doses of Puerariae Radix above $60 \mathrm{~g}$.

In addition, the present data show that the major consumers of CDG-CHPs were aged between 20 and 49 years. Little is known about phytoestrogenic herbs and their potential interactions with the endocrine system, and therefore special attention should be drawn to the herbal formulae containing phytoestrogenic herbs, especially the prescriptions for female patients of reproductive age suffering from respiratory diseases.

According to CM theory [12], Puerariae Radix can dispel exterior cold syndrome of the excessive type and alleviate muscle aches in the neck and back caused by cold constriction. Previous in vitro studies have indicated that Puerariae Radix is rich in coumestrol, genistein and daidzein as soya, which may reduce cancer cell viability and induce apoptosis $[13,14]$. And, epidemiological studies also suggested that exposure to relatively high concentrations of phytoestrogens early in life may decrease the prevalence of cancer development later in life $[15,16]$. CM doctors should observe the potential effects of phytoestrogenic herbs on consumers' endocrine systems proactively at the early stage to avoid any consequences of chronic $\mathrm{CM}$ therapy administration.

Our study has two limitations. First, the NHI system only reimburses CHPs, and decoctions are not included and cannot be generalized for their usage. In addition, this study did not include Chinese herbal remedies and health foods containing herbs that can be directly purchased from CM pharmacies. Thus, the frequency of phytoestrogenic herb consumption might be underestimated. Because the NHIRD collects all prescription information prospectively, we can rule out the possibility of a recall bias concerning intake dosages and different types of herbal prescriptions. In addition, as the rate of insured individuals in the NHIRD has consistently exceeded 96\% since 1997, we can exclude the possibility of a selection bias. Therefore, the estimated prevalence rate presented here is close to the true use of CGD-CHPs by female beneficiaries in Taiwan. Another limitation is that only herbs containing coumestrol, genistein and/or daidzein were included in the study, meaning that there must be caution in generalizing these findings to use of phytoestrogens among the female population.

\section{Conclusion}

This study shows that women who sought medical treatment from CM doctors for relief of respiratory discomfort had a high possibility of exposure to phytoestrogenic herbs. Safety issues related to the female endocrine system should be a priority for future research.

\section{Abbreviations}

CM: Chinese medicine; CHP: Chinese herbal products; CGD-CHPs: Chinese herbal products containing coumestrol, genistein and/or daidzein; $\mathrm{NHI}$ : National Health Insurance; NHIRD: national health insurance research database; CCMP: Committee on Chinese Medicine and Pharmacy; ICD-9CM: International Classification of Diseases, 9th Revision, Clinical Modification.

\section{Competing interests}

The sponsors of the study had no role in the study design, data collection, data analysis, data interpretation, or writing of the report. All authors had financial support from the Committee on Chinese Medicine and Pharmacy of the Department of Health (Taiwan), and National Health Research Institutes (Taiwan) for the submitted work; no financial relationships with any organizations that might have an interest in the submitted work in the previous 5 years; and no other relationships or activities that could appear to have influenced the submitted work.

\section{Authors' contributions}

CTW, JNL, and JDW designed the study. CTW and JNL acquired the data. CTW, JNL, and JDW analyzed the data. CTW and JNL wrote the manuscript. JNT, HST, and JDW revised the manuscript. JDW provided technical advice. All authors read and approved the final version of the manuscript.

\section{Acknowledgments}

The study was funded by two grants: (CCMP96-RD-021 and CCMP97-RD-110) from the Committee on Chinese Medicine and Pharmacy of the Department of Health (Taiwan), and a grant (NRICM-9903) from the National Health Research Institutes (Taiwan). We would like to thank Tung-Hu Tsai, ChangHsing Lee, and Yao-Hsu Yang for their professional assistance with the statistical application.

\section{Author details}

${ }^{1}$ Department of Chinese Medicine, Linsen (Chinese Medicine) Branch and Chinese Medicine Clinic Center, Taipei City Hospital, Taipei, Taiwan. ${ }^{2}$ Institute of Traditional Medicine, School of Medicine, National Yang-Ming University, Taipei, Taiwan. ${ }^{3}$ Department of Mathematical Sciences, National Chengchi University, Taipei, Taiwan. ${ }^{4}$ Department of Obstetrics and Gynecology and Department of Chinese Medicine, Yangming Branch, Taipei City Hospital, Taipei, Taiwan. ${ }^{5}$ Department of Obstetrics and Gynecology, Zhongxing Branch, Taipei City Hospital, Taipei, Taiwan. 'Department of Public Health, College of Medicine, National Cheng Kung University, Tainan, Taiwan. ${ }^{7}$ Departments of Occupational and Environmental Medicine and Internal Medicine, National Cheng Kung University Hospital, Tainan, Taiwan.

Received: 19 December 2011 Accepted: 9 October 2012

Published: 16 October 2012

\section{References}

1. Humfrey CD: Phytoestrogens and human health effects: weighing up the current evidence. Nat Toxins 1998, 6:51-59.

2. Cassidy A: Potential risks and benefits of phytoestrogen-rich diets. Int J Vitam Nutr Res 2003, 73:120-126.

3. Limer JL, Parkes AT, Speirs V: Differential response to phytoestrogens in endocrine sensitive and resistant breast cancer cells in vitro. Int $J$ Cancer 2006, 119:515-521.

4. Golden RJ, Noller KL, Titus-Ernstoff L, Kaufman RH, Mittendorf R, Stillman R, Reese EA: Environmental endocrine modulators and human health: an assessment of the biological evidence. Crit Rev Toxicol 1998, 28:109-227.

5. Takeuchi S, Takahashi T, Sawada Y, lida M, Matsuda T, Kojima H: Comparative study on the nuclear hormone receptor activity of various phytochemicals and their metabolites by reporter gene assays using Chinese hamster ovary cells. Biol Pharm Bull 2009, 32:195-202. 
6. Harris DM, Besselink E, Henning SM, Go VL, Heber D: Phytoestrogens induce differential estrogen receptor alpha- or Beta-mediated responses in transfected breast cancer cells. Exp Biol Med (Maywood) 2005, 230:558-568.

7. Lee SH, Jung BH, Kim SY, Chung BC: Determination of phytoestrogens in traditional medicinal herbs using gas chromatography-mass spectrometry. J Nutr Biochem 2004, 15:452-460.

8. Yang YH, Chen PC, Wang JD, Lee CH, Lai JN: Prescription pattern of traditional chinese medicine for climacteric women in taiwan. Climacteric 2009, 12:541-547.

9. Chou SY, Liu JT, Hammitt JK: National health insurance and precautionary saving: evidence from taiwan. J Health Econ 2003, 87:1873-1894.

10. International publications regarding the use of national health insurance database taiwan. http://www.nhri.org.tw/nhird/talk_07.htm.

11. The national health insurance statistics - beneficiaries profile. http://www.nhi gov.tw/english/webdata.asp? menu $=11 \&$ menu $i d=290 \&$ Cmenu $I D=302 \&$ webdata id $=2974$

12. Cai YM, He Y, Qiu T, Zou J, Sun DP, Peng QH, Jia RX, Zhao HR: Research on frequency of application with modern chinese herbal medicine. Chin J Integr Med 2011, 17:64-70.

13. Lin YJ, Hou YC, Lin CH, Hsu YA, Sheu JJ, Lai CH, Chen BH, Lee Chao PD, Wan L, Tsai FJ: Puerariae radix isoflavones and their metabolites inhibit growth and induce apoptosis in breast cancer cells. Biochem Biophys Res Commun 2009, 378:683-688.

14. Yu Z, Li W: Induction of apoptosis by puerarin in colon cancer HT-29 cells. Cancer Lett 2006, 238:53-60.

15. Caan BJ, Natarajan L, Parker B, Gold EB, Thomson C, Newman V, Rock CL, Pu M, Al-Delaimy W, Pierce JP: Soy food consumption and breast cancer prognosis. Cancer Epidemiol Biomarkers Prev 2011, 20:854-858.

16. Messina M, Hilakivi-Clarke L: Early intake appears to be the key to the proposed protective effects of soy intake against breast cancer. Nutr Cancer 2009, 61:792-798.

doi:10.1186/1749-8546-7-22

Cite this article as: Lai et al.: Prescription profile of Chinese herbal products containing coumestrol, genestein, and/or daidzein among female users: an analysis of national health insurance data in Taiwan between 1997 and 2007. Chinese Medicine 2012 7:22.

\section{Submit your next manuscript to BioMed Central and take full advantage of:}

- Convenient online submission

- Thorough peer review

- No space constraints or color figure charges

- Immediate publication on acceptance

- Inclusion in PubMed, CAS, Scopus and Google Scholar

- Research which is freely available for redistribution

Submit your manuscript at www.biomedcentral.com/submit
() Biomed Central 